\title{
Markers of clinical utility in the differential diagnosis and prognosis of prostate cancer
}

\author{
Glen Kristiansen \\ Institute of Pathology of the University Hospital Bonn, Bonn, Germany
}

\begin{abstract}
Molecular diagnostics is a rapidly evolving area of surgical pathology, that is gradually beginning to transform our diagnostical procedures for a variety of tumors. Next to molecular prognostication that has begun to complement our histological diagnosis in breast cancer, additional testing to detect targets and to predict therapy response has become common practice in breast and lung cancer. Prostate cancer is a bit slower in this respect, as it is still largely diagnosed and classified on morphological grounds. Our diagnostic immunohistochemical armamentarium of basal cell markers and positive markers of malignancy now allows to clarify the majority of lesions, if applied to the appropriate morphological context (and step sections). Prognostic immunohistochemistry remains a problematic and erratic yet tempting research field that provides information on tumor relevance of proteins, but little hard data to integrate into our diagnostic workflow. Main reasons are various issues of standardization that hamper the reproducibility of cut-off values to delineate risk categories. Molecular testing of DNA-methylation or transcript profiling may be much better standardized and this review discusses a couple of commercially available tests: The ConfirmDX test measures DNA-methylation to estimate the likelihood of cancer detection on a repeat biopsy and may help to reduce unnecessary biopsies. The tests Prolaris, OncotypeDX Prostate, and Decipher all are transcript tests that have shown to provide prognostic data independent of clinico-pathological parameters and that may aid in therapy planning. However, further validation and more comparative studies will be needed to clarify the many open questions concerning sampling bias and tumor heterogeneity.

Modern Pathology (2018) 31, S143-S155; doi:10.1038/modpathol.2017.168
\end{abstract}

In the western world prostate cancer still is the most common non-cutaneous malignant tumor in men with an expected incidence of 161360 new cases in 2017 for the USA alone. ${ }^{1}$ Even though a wider medical awareness of prostate cancer, its diagnosis and treatment arose late with the beginning of the twentieth century, its presence can be documented in archeological findings. The oldest documented case of a metastasized prostate cancer is that of a scythian king, buried 2700 years ago in northern siberia, whose grave was excavated in 2003. The well preserved skeleton showed focal signs of osseous thickening as is commonly seen in osteoplastic metastases. A subsequent molecular analysis eventually demonstrated prostate-specific antigen (PSA) in these lesions, confirming that this individual had suffered from metastasized prostate cancer. ${ }^{2}$ This case not only confirms that prostate cancer has

Correspondence: Professor G Kristiansen, MD, Institute of Pathology of the University Hospital Bonn, Sigmund-Freud-Str. 25, 53127 Bonn, Germany.

E-mail: Glen.Kristiansen@ukbonn.de

Received 31 August 2017; revised 2 November 2017; accepted 3 November 2017 accompanied (male) mankind for a while already, but it also illustrates the general value of biomarkers, that carry additional information, help to clarify assumptions and ideally turn them into facts. In comparison to other, more aggressive tumors, prostate cancer has a relatively low mortality rate, ranked only third according to current expectations, after lung and colon cancer. ${ }^{1}$ Its high incidence, low mortality, divergent attitudes about the value of PSA screening constitute a significant clinical problem in individualized patient care. ${ }^{3,4}$ To date, it is still unclear which type of therapy is most appropriate in which setting and especially cases with low risk disease are at high risk of overtreatment. ${ }^{5}$ Even though this has meanwhile been acknowledged and this has helped to promote active surveillance as a novel strategy to defer curative therapy with its iatrogenic morbidity, it is still unclear how to rationally stratify patients for the diverse therapy options. Clearly, new biomarkers are needed to overcome this problem. Diagnostic biomarkers may aid to confirm a cancer diagnosis in tiny lesions or even retrieve diagnostic information from apparently benign tissues. This would help to reduce unnecessary re-biopsies or inform us about the necessity for 
an early re-biopsy. Another important and competing development that ought to be mentioned here is multiparametric MRI scanning for improved radiological detection and classification of prostate cancer. It is expected that this technique will eventually lead to a reduced biopsy rate, as it also shows promise to allow for a non-invasive classification of prostate cancer aggressiveness. However, it is currently unclear, whether new tissue-based biomarkers or better radiology will predominate therapy planning in the future. ${ }^{6,7}$

Following the initial histological diagnosis of prostate cancer, even more relevant are prognostic biomarkers that classify the biologic potential of a tumor better than histology alone. This would help answering the pressing question, who can safely be spared active therapy for some time and who would benefit from immediate treatment. The molecular definition of significant and lethal prostate cancer is still a highly relevant issue, that has no definitive answer yet. The even more refined demand for biomarkers that specifically predict the long-term outcome of surgery vs radiation therapy will probably not be satisfied in the near future, but is another clinically important research area. Prognostic and predictive biomarkers will also become more relevant in later stages of prostate cancer, when it comes to prognosticate the onset of castration resistance or the likelihood of drug response in castrationresistant prostate cancer (CRPC).

\section{Diagnostic immunohistochemistry}

In contrast to other areas of surgical pathology, specifically haemato- and lymphoma pathology with its endless arsenal of CD-antigens necessary for correct tumor typing, prostate cancer immunohistochemistry appears relatively primitive: with a basal cell marker, Alpha-Methylacyl-CoA Racemase (AMACR), and PSA alone most diagnostic questions can be sufficiently mastered. Still, the correct interpretation of these markers is highly dependent on the morphological context, probably more so than in lymphoma diagnostics. Therefore, it is comforting to know additional marker options, if necessary. But irrespective of the choice of markers, one should bear in mind the (three) dimensionality of prostatic lesions, which necessitate serial sections to appreciate. In my experience to rely on a single immunohistochemistry section of a small doubtful lesion is a negligent approach, so three levels are a recommended minimum.

\section{Basal cell markers}

The absence of basal cells has long been recognized as a defining criterium of acinar prostate cancer. ${ }^{8}$ With the introduction of immunohistochemistry as a new tool for surgical pathologists in the 70s of last century, chromogenic labeling of basal cells on paraffinized tissue sections soon became feasible. It caught ground rapidly and still constitutes the cornerstone of diagnostic immunohistochemistry in prostate cancer. ${ }^{9,10}$ Following the high molecular weight cytokeratins (CK34 $\beta \mathrm{E} 12$, CK5/6, or CK5/14), the p53 homolog p63 was introduced as an alternative basal cell marker that displayed a crisp nuclear staining and which remains a preferred choice till today. ${ }^{11}$ In my experience, p63 is slightly more sensitive in labeling basal cells from glands of the transitional zone than basal cell cytokeratins, which may be a fixation artifact and which may also be laboratory dependent. ${ }^{12}$ More recently, the p63 isoform $\Delta \mathrm{Np} 63$ (p40), which features a transcriptionally inactive $\Delta \mathrm{N}$ domain, has been suggested as an alternative basal cell marker. Even though p40 shows a minimally lower rate of false-positive labeling of carcinomas, most authors agree that the differences seen in real life situations are negligible. ${ }^{13,14}$ It is, however, important to realize that every new diagnostic tool introduces new pitfalls and p63-/or p40-positive carcinomas are a good example of this. ${ }^{15}$ As they are usually negative for high molecular weight cytokeratins, an additional immunstain can clarify doubtful cases.

\section{Positive markers of malignancy}

To increase the specificity of a cancer diagnosis further beyond basal cell immunohistochemistry has long been desired. AMACR has been the first marker that entered common practice, following its identification as a commonly overexpressed transcript in prostate cancer nearly two decades ago ${ }^{16-19}$ ). AMACR immunohistochemistry can be immensely helpful especially in small foci on needle biopsy, but again it is not perfect. First, its expression in prostate cancer is heterogenous and it may even be completely absent in $5 \%$ of cases. Also, hyperplastic glands but more important typical prostate cancer mimicks such as partial atrophy or adenosis may display weak to moderate levels of AMACR. ${ }^{20}$ Finally, highgrade prostatic intraepithelial neoplasia (PIN) as the best documented prostate cancer precursor lesion often shows AMACR overexpression, which limits the value of this marker to discriminate assumedly non-invasive high-grade PIN outpuchings from glands of invasive adenocarcinoma. ${ }^{21}$ It is also important to note that AMACR cannot be used as a lineage marker, as it is not prostate-specific, but seen in a wider variety of tumors including renal, urothelial, and colon cancer. ${ }^{22-25}$ All this underscores the importance of the morphological context in which prostate immunohistochemistry has to be placed. Nonetheless, the combination of a basal cell marker with AMACR (eg, p63/AMACR) is a very powerful first-step immunostain (Figure 1a).

Some alternatives to AMACR have been suggested, that may prove helpful where AMACR fails. Fatty 

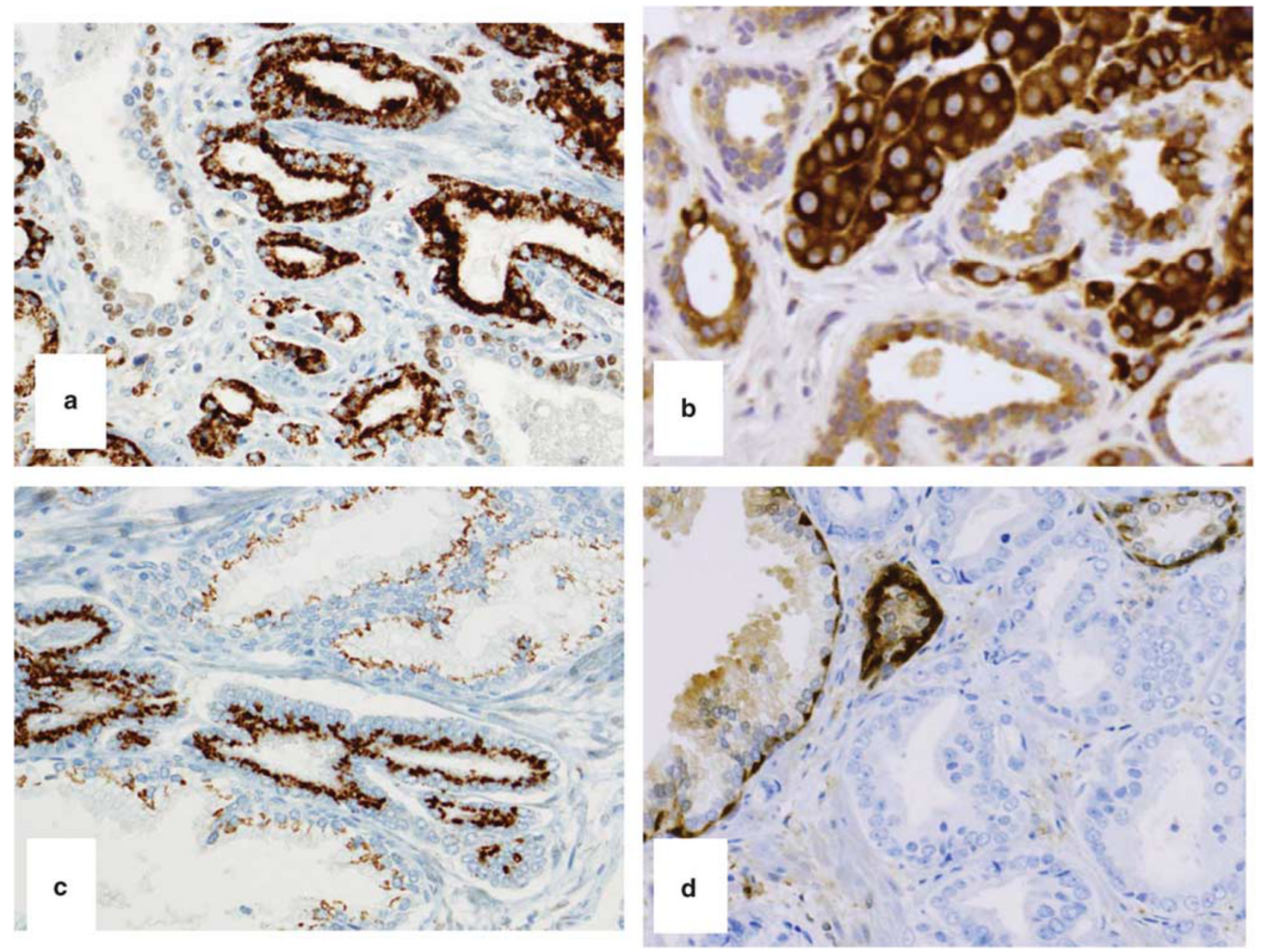

Figure 1 Positive markers of malignancy in prostate cancer. (a) p63/AMACR showing the typical slightly granular expression of AMACR in striking contrast to adjacent benign glands. (b) FASN is also widely expressed in normal tissues, but is upregulated in tumor glands. (c) GOLM1 is a bit more difficult to read, as it necessitates to identify unequivocally benign glands in the vicinity. However, the characteristic Golgi pattern confirms proper staining and the contrast of the malignant glands is stark. (d) GST-pi usually shows a moderate labeling of basal cells and a slightly weaker positivity in benign secretory epithelium. The glands of the invasive carcinoma (lower right corner) are completely devoid of immunoreactivity.

acid synthase (FASN) is also overexpressed in prostate cancer and a gland to gland comparison of clearly benign glands with the suspicious glands under scrutiny may be helpful (Figure 1b). As FASN is already positive in normal epithelia (more so than AMACR), this comparative approach is advised. If used accordingly, FASN will positively label 93\% of carcinomas correctly and especially the majority of AMACR-negative cases will be picked up. ${ }^{26}$ This compares to the performance of GOLM1 (GOLPH2, GP73) that is also overexpressed in prostate cancer and may contribute to an affirmative cancer diagnosis. ${ }^{27-29}$ GOLM1 stainings typically show a distinctive Golgi pattern, but again the comparison to adjacent clearly benign glands is crucial, as GOLM1 appears to be rather ubiquituously expressed (Figure 1c). Again, it is also not prostate-specific but seen in hepatocellular carcinoma, renal cancer, seminoma, and other tumors. ${ }^{30-32}$

As the discovery of the TMPRSS2-ERG-Translocation, which occurs in $\sim 50 \%$ of prostate carcinomas and which is highly prostate cancer-specific, its diagnostic role has been discussed (excellently reviewed by Dr. Berg ${ }^{33}$ ). The introduction of specific ERG antibodies have enabled to detect ERG overexpression as a surrogate marker of TMPRSS2-ERG translocation, but naturally this does not change its positivity rate of $50 \%$, which is limiting its diagnostic value. ${ }^{34}$

Not exactly a positive marker but more an additional negative marker of malignant secretory epithelium is Glutathione-S-transferase-pi (GST-pi). Promoter methylation of GST-pi commonly occurs in prostate cancer and the concomitant downregulation of GST-pi protein expression can in principle be diagnostically utilized. ${ }^{35}$ According to our own data, $96 \%$ of cancer cases show a complete loss of GST-pi immunoreactivity (Figure 1d), however, adjacent normal tissue also showed a loss in $25 \%$ of cases, limiting its diagnostic value. ${ }^{36}$

As stressed before, for diagnostic use it is advisable to stick to those markers that the wealth of our 
literature is based on and that were recommended at the 2013 consensus conference of the International Society of Urological Pathology (ISUP). ${ }^{37}$

\section{Confirmation of prostatic origin}

Determining the origin of metastases has always been an important task of pathologists to guide the clinician in choosing a tumor-specific therapy. PSA has become the dominant prostate cancer serum marker ${ }^{38,39}$ and it can be detected by immunohistochemistry in tissues as well. PSA still is regarded as a highly specific marker of prostatic origin, but due to its decreased expression in higher grade or metastatic tumors, which was already noted in initial studies, its sensitivity is limited, which necessitates the use of additional markers. Prostate-specific alkaline phosphatase (PSAP) has long been recommended by some, but it is highly correlated with PSA expression and like PSA it tends to get lost with tumor progression, hampering its value. ${ }^{40-42}$

Prostein, coded by the SLC45A3 gene, is another marker that is highly specific for prostatic origin. ${ }^{43,44}$ Even though its expression decreases slightly with tumor progression, in combination with PSA it can be helpful to identify prostatic origin (Figure 2a). ${ }^{45,46}$ An alternative marker found to be highly specific for prostate tissues is the homeobox gene NKX3.1, which shows a nuclear staining (Figure 2b). ${ }^{47,48}$ At the 2013 consensus conference of the International Society of Urological Pathology (ISUP), it was recommended to use immunohistochemistry for PSA, NKX3.1 and Prostein to ascertain a prostatic origin in doubtful cases. ${ }^{37}$ In many diagnostic settings, a combination of markers will be required to clarify tumor origin. ${ }^{49}$ Recently, the homeobox protein Hox-B13 (HOXB13), introduced as a prostate cancer marker by Edwards et al, has been suggested as diagnostically useful and has been recommended as a prostate-specific marker; ${ }^{50-54}$ however, its additional value especially over NKX3.1 is under dispute, as the author's own data suggest, finding HOXB13 positivity in only $60.4 \%$ of cases (compared to $100 \%$ for NKX3.1). ${ }^{42}$

\section{Prognostic immunohistochemistry}

To cover the wealth of prognostic immunohistochemical markers that have been proposed for prostate cancer is beyond this review and a focus will be layed on Ki-67, PTEN, and ERG. But first, is immunohistochemistry as the most common technical platform in prognostic biomarker studies really sufficient for this task? Does it yield reproducible results? Bearing in mind, that this technique is a qualitative method to detect epitopes by means of specific antibodies, this question appears rethorical. To verify the validity of prognostic immunomarkers, we assembled a typical set of 28 prognostic biomarkers from the literature. ${ }^{55}$ For validation of these markers, a tissue micro array (TMA) was used that sampled 238 radical prostatectomy cases with one core each. The results were surprising: on univariate Kaplan-Meier analysis, only 11 markers showed a significant prognostic value but even worse, on multivariable Cox analysis only 4 markers (AKT1, stromal AR, EZH2, and PSMA) remained significant. This may indicate that our study was either flawed or underpowered. This is possible, but the same would be true for the original studies, as the number of cases analyzed is comparable. One could also argue that a single core of tumor tissue cannot sufficiently represent a tumor, hence this study was unable to reproduce the prognostic values found by others. Conversely, one could argue that a prognostic biomarker of clinical value should be resistant to sampling issues. It appears likely, that in the past many biomarker studies have been slightly overoptimistic and also had deficits in the statistical design (missing training/testing sets). The retrospective observation of a prognostic value thus does not necessarily allow a prospective application, which makes it so difficult to establish an immunohistochemical prognostic test for patient care.

Even biomarkers like Ki-67, that have been extensively validated, have not entered clinical practice so far. ${ }^{56}$ More than 20 studies conclude that higher rates of Ki-67 immunoreactivity indicate more aggressive disease (reviewed in ref. 57). A metaanalysis is still difficult, as these studies use different cohort types, different statistical endpoints, different cut-off values (ranging from 2.4 to $26 \%$ ) and finally construct multivariate models differently. Clearly, a publication of the underlying raw datasets would enable post-publicatory peer review and make metaanalyses feasible. Editors of scientific journals should be more demanding in this respect in the future and to establish an international standard of (supplementory) data deposition would be a highly desirable next step. Also the REMARK guidelines, that have assembled key factors for successful biomarker establishment should be more intensively promoted, as their recognition and application appears to catch on only slowly. ${ }^{58,59}$

The technical side of immunostaining needs to be better standardized as well. The preanalytical influences are significant and may differently impact on different epitopes: time to fixation, time in fixation, type of fixative, embedding, and storage conditions. Among relevant technical analytical influences are sectioning, slide baking, type of antigen retrieval, choice of detection system, and chromogen. Even more relevant is interoberver variability in reading immunohistochemistry slides (see Figure 3-how many cells are positive?). Even among expert laboratories the variability in reading Ki-67 scores is astounding. Polley et al sent a centrally Ki-67 stained TMA with 100 breast cancer cases to eight laboratories. ${ }^{60}$ The decentral evaluations revealed a remarkable spread of median Ki-67 scores, ranging 

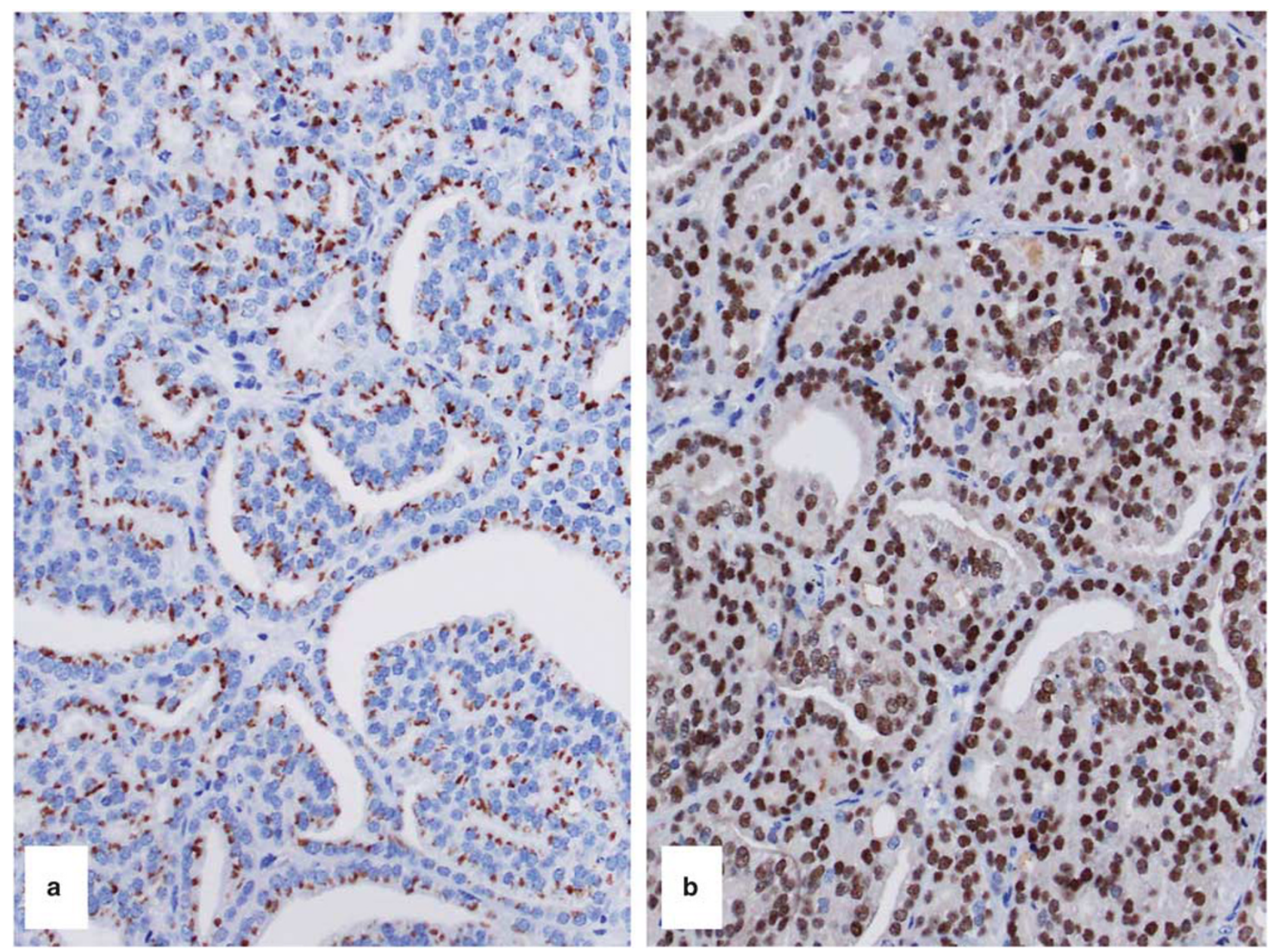

Figure 2 Immunohistochemistry in a prostate cancer metastasis. (a) Typical example of positive Prostein immunoreactivity with the Golgi-like staining in the cytoplasm. (b) NKX3.1 displays a strong nuclear staining and is generally better retained than Prostein during tumor progression.

from 10 to $28 \%$. This range even increased (5-33\%) when each laboratory stained their slide themselves. How can we standardize immunohistochemistry further? An exploratory checker board titration experiment with Ki-67 demonstrated that the dilution titrates the proliferative fraction-this renders absolute thresholds of Ki-67 positivity for diagnostic purposes doubtful, at least with current techniques. Maybe standardized positive controls can be helpful. Another factor to consider in prognostic biomarkers is the minimum amount of tumor tissue necessary to allow for a reliable test result. It is likely that this size of the region of interest (ROI) varies from biomarker to biomarker and should be individually determined. Christgen et al ${ }^{61}$ evaluated the impact of ROI size on Ki-67 positivity in breast cancer using computer assisted image analysis and they found the Ki-67 labeling index to depend on ROI size. Starting from $400+$ cells they found labeling indices to become stable. Denkert et $a l^{62}$ simulated sampling bias in a computer model, which confirmed a relevant impact of sampling size on stability of measurements. Little is known about the ROI size necessary for reliable Ki-67 determinations in prostate cancer. In our own study a minimum of $2.5 \mathrm{~mm}^{2}$ was necessary to demonstrate a prognostic value of Ki-67 in a patient subgroup. ${ }^{63}$ If this was independently confirmed, it would imply that Ki-67 is useless to assess tumor aggressiveness in minute $(<2.5 \mathrm{~mm})$ cancer infiltrates on biopsy.

Alterations of the tumor suppressor gene Phosphatase and Tensin Homolog (PTEN) belong to the early and common alterations in prostate cancer. ${ }^{64} \mathrm{In}$ mice models PTEN loss in conjunction with other alterations (especially ERG overexpression) contributes to carcinogenesis. ${ }^{65,66}$ Many studies convene in the recognition of a worse prognosis of prostate carcinomas with PTEN loss/diminished PTEN expression. ${ }^{67-71}$ The largest study to date found a good concordance of genomic loss (as detected by FISH) with PTEN protein loss (detected by immunohistochemistry) but recommended both techniques to detect PTEN alterations. ${ }^{72}$ According to Trock et al, ${ }^{73}$ PTEN loss may predict adverse pathology in cases with Gleason score $3+3=6$ carcinoma in prostate biopsies, but further validation is needed. As with other biomarkers, PTEN expression displays a considerable intratumoral heterogeneity. ${ }^{74}$ Still, 


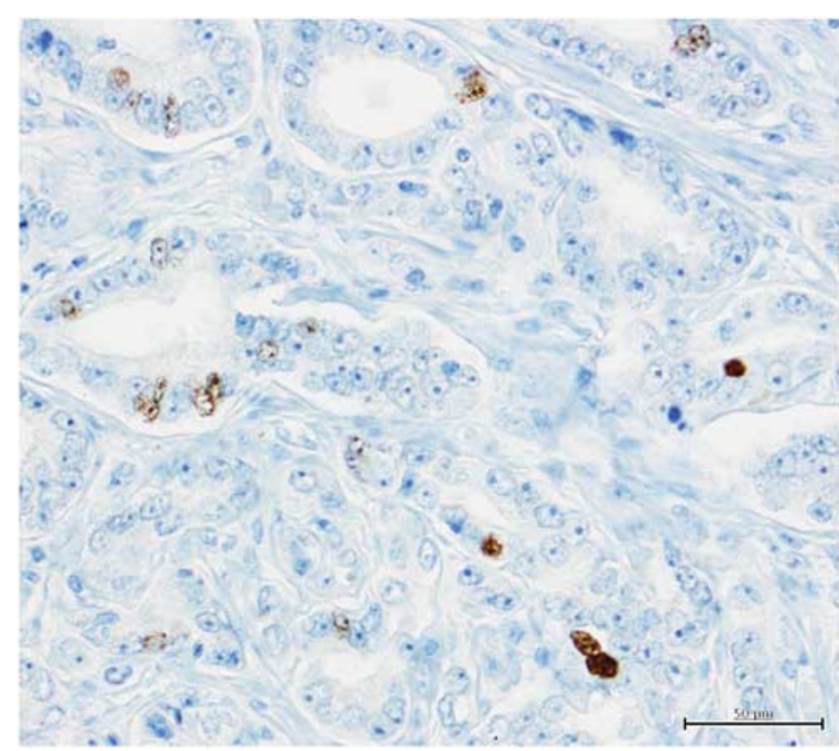

Figure 3 Ki-67 Immunohistochemistry-range of positivity. How many cells would you count as positive? If counted conservatively, only strongly labeled nuclei would be called positive, resulting in $4 \%$. Alternatively, one might include also the moderately or weakly stained nuclei, resulting in 8 or even $16 \%$.

PTEN has a significant role not only during prostatic carcinogenesis, but may also serve as a predictive marker for response to advanced anti-androgenic therapy (ie, abiraterone) in later stages of this disease. ${ }^{75}$

The surprising discovery of TMPRSS2-ERG as the most common genomic translocation in primary prostate cancer (seen in $\sim 50 \%$ of cases) more than a decade ago has given major impulses to prostate cancer research. ${ }^{76}$ This alteration, that leads to regulation of potentially oncogenic factors of the E26 transformation-specific (ETS) transcription factor family by androgen signaling broadened our comprehension of prostatic carcinogenesis. The prognostic value of the TMPRSS2-ERG gene fusion is still unclear and the data is apparently conflicting. In most watchful waiting cohorts, TMPRSS2-ERGpositive cases are associated with a worse prognosis. ${ }^{77,78}$ This matches the observation, that ERG positivity as detected by IHC has been found prognostic in an active surveillance cohort, indicating failure of surveillance. ${ }^{79}$ However, following radical prostatectomy, the vast majority of studies does not show a prognostic value of the TMPRSS2ERG fusion or ERG overexpression. ${ }^{80-84}$ The high intratumoral heterogeneity of ERG expression may contribute to this. ${ }^{85}$ As of yet, the clinical value of TMPRSS2-ERG gene fusion or ERG overexpression (as a surrogate marker for the former) is not fully determined, but many biomarker studies have highlighted the modifier character of this alteration, defining a subgroup of prostate cancer patients in which another biomarker is particularly prognostic (eg, Ki-67 or PTEN in ERG-negative cases). ${ }^{63,86}$

\section{Molecular tests in prostate cancer}

So far, molecular testing has no role in routine work up of newly diagnosed prostate cancer cases. In general, molecular alterations of the tumor may be diagnostic, prognostic or predictive and any biological level can be assessed with modern tools. DNA sequencing of prostate cancer has no role yet, however, the introduction of PARP inhibition in late stage castration-resistant prostate cancer may necessitate sequencing to detect DNA repair defects and thus induce a change of practice in the nearer future. ${ }^{87}$ In the following, I will focus on DNAmethylation as an example for a diagnostic test and the application of transcript signatures as prognostic markers.

\section{DNA-methylation}

Promoter methylation is one of the most common molecular alterations observed in cancer in general and also in prostate cancer. ${ }^{88,89}$ Also, the stability of DNA makes it an attractive analyte, that can easily be tested from formalin-fixed paraffin embedded (FFPE) tissues with modern methods. ${ }^{90}$ For prostate cancer, a variety of genes has been suggested as prognostic methylation marker in smaller studies with PITX2 being most extensively verified so far. ${ }^{91-99} \mathrm{~A}$ commercialized diagnostic test, that measures gene methylation in tissues of an initial negative biopsy to estimate the likelihood of a cancer diagnosis on subsequent biopsy is the ConfirmDX test ( $\mathrm{MDx}$ Health). The histological demonstration of carcinoma is still the diagnostic gold standard to allow for curative therapy. The relatively high number of negatives seen under conventional regimens of transrectal ultrasound-guided biopsies pose a problem for patients, clinicians, and pathologists alike. ${ }^{100}$ This dilemma may improve with more widespread use of MRI guided biopsy, which also appears to have a welcome bias to pick up higher grade tumors more reliably. ${ }^{101}$ Apart from serum PSA levels and its velocity, the only widely accepted histological indicators of increased cancer likelihood in cancer-negative biopsies are multifocal high PIN and atypical small acini (ASAP), that may represent small foci of cancer, but that cannot be diagnosed with certainty. ${ }^{102}$ In this situation, a molecular tool to guide the decision on the timing of follow-up biopsy may prove valuable. The ConfirmDX test measures promoter methylation of the three genes GSTP1, APC, and RASSF1, all known to be commonly hypermethylated in prostate cancer, using a quantitative methylation specific polymerase chain reaction assay. A prior study by Trock et al ${ }^{103}$ had shown promising predictive properties of APC and GSTP1 alone in a set of 86 patients with a negative initial biopsy. An expanded analysis of APC, GSTP1 and RASSF1 in a cohort of 498 patients with an initial negative biopsy and a re-biopsy within 
30 months demonstrated a negative predictive value of $90 \%$ (MATLOC study). More, the multivariate analysis demonstrated this marker panel as an independent risk factor for the histological detection of carcinoma on re-biopsy. ${ }^{104}$ A subsequent validation study encompassing 350 patients with negative primary biopsies from five urological centers (DOCUMENT trial) in the United States confirmed this negative predictive value (88\%). ${ }^{105}$ This methylation score even outperformed the predictive value of a histological diagnosis of ASAP by a small degree. It remains to be seen, if molecular testing of negative biopsies or new approaches of MRI imaging will prove more powerful and cost effective to reduce unnecessary re-biopsies.

\section{Prognostic transcript signatures}

With the advent of high-throughput expression profiling techniques, numerous groups have sought to establish new molecular markers and prognostic signatures for prostate cancer on transcript level. ${ }^{106-112}$ Even though the earlier studies have contributed a lot of data for further research, more extensive validation of prognostic signatures was not published. Sboner et al ${ }^{113}$ had conducted expression profiling (6100 transcripts) from FFPE tissue of a high quality watchful waiting cohort $(n=281)$ with long-term follow-up data and applied state of the art statistical methods including a training and a testing cohort to establish a signature of lethal cancer. However, their attempt failed. The authors realized, that none of their models outperformed clinico-pathological parameters including the Gleason score and they also hypothesized that tumor heterogeneity was a major issue in prostate cancer sampling. Nonetheless, transcript profiling allows standardization of measurements in contrast to (contemporary) immunohistochemistry by normalizing the target gene expression with housekeeping genes and this makes this platform very interesting for biomarker development. ${ }^{114,115}$

In the following, three examples of commercialized prognostic tests will be discussed: Prolaris (Myriad Genetics), OncotypeDX Prostate (Genomic Health), and Decipher (GenomeDX Biosciences). Historically, all three signatures were constructed from transcript profiles of retrospectively characterized cohorts, mainly of radical prostatectomy (RP) origin. The construction of the Oncotype signature also involved expression data from biopsies (with adverse pathology on RP as endpoint) to consider sampling biases and tumor heterogeneity. The Decipher test initially aimed to prognosticate outcome following radical prostatectomy, whereas the other tests claimed to be helpful in earlier therapy planning. By now is it obvious that all three tests carry prognostic information, independent of clinico-pathological parameters, that may be helpful in different stages of the disease (Table 1). ${ }^{115}$ Clearly, it will need further studies to clarify the pros and cons of these tests in various stages of prostate cancer.

\section{Prolaris}

Increased cellular proliferation is a hallmark of cancer and also a prognostic factor in virtually all malignant tumors. ${ }^{116}$ Cuzick et $a l^{117}$ have constructed a proliferation signature that finally consisted of 31 cell cycle progression (CCP) transcripts (FOXM1, CDC20, CDKN3, CDC2, KIF11, KIAA0101, NUSAP1, CENPF, ASPM, BUB1B, RRM2, DLGAP5, BIRC5, KIF20A, PLK1, TOP2A, TK1, PBK, ASF1B, C18orf24, RAD54L, PTTG1, CDCA3, MCM10, PRC1, DTL, CEP55, RAD51, CENPM, CDCA8, and ORC6L), having screened 126 CCP genes that were selected from the Gene Expression Omnibus Database. A selection criterium was applicability to FFPE tissues and gene expression was normalized by measurement of another 15 housekeeper genes. The resultant data was compiled into a CCP score, which was prognostic of outcome in a radical prostatectomy cohort ( $n=366$; BCR as endpoint) and a watchful waiting cohort $(n=337$; cancer-specific death as endpoint). In both settings, the CCP score was demonstrated as a multivariate prognostic factor. Following studies of the same group analyzed the CCP score in a conservatively managed needle biopsy cohort and validated it as the strongest independent predictor of cancer death in their multivariate models. ${ }^{118,119}$ Further validation of the prognostic value of the CCP score followed in independent prostatectomy and radiation therapy cohorts. ${ }^{120-122}$ Oderna et al ${ }^{123}$ compared the CCP score to EAU clinical risk scores and Ki-67 scores

Table 1 Overview of molecular tests for prostate cancer

\begin{tabular}{lllll}
\hline Test name & Rationale of the signature & Clinical setting & $\begin{array}{l}\text { Possible application according } \\
\text { to manufacturer }\end{array}$ & $\begin{array}{c}\text { Costs (USD; 2015) current } \\
\text { prizes may vary }\end{array}$ \\
\hline Prolaris & Measuring proliferation & $\begin{array}{l}\text { Primary positive biopsy/ } \\
\text { following local therapy }\end{array}$ & $\begin{array}{l}\text { Helpful prior and after local } \\
\text { therapy }\end{array}$ & $\begin{array}{l}\text { Helpful prior to local therapy } \\
\text { OncotypeDX }\end{array}$ \\
$\begin{array}{l}\text { Decipher } \\
\text { or poor outcome pathology } \\
\text { Predicting metastasis }\end{array}$ & $\begin{array}{l}\text { Primary positive biopsy } \\
\text { Following local therapy }\end{array}$ & $\begin{array}{l}\text { Helpful following local } \\
\text { therapy }\end{array}$ & 4250 \\
\hline
\end{tabular}


and confirmed that the CCP score adds information beyond traditional clinical variables. A recent multicenter study evaluated the CCP in a cohort of 236 low risk patients, according to definitions of the National Comprehensive Cancer Network (NCCN). The results suggested that the CCP score might aid in identifying patients suitable for an active surveillance strategy. ${ }^{124} \mathrm{~A}$ real prospective validation of the CCP score in an active surveillance setting has not been performed yet.

\section{OncotypeDX prostate}

The OncotypeDX Prostate assay is based on a signature of 12 genes relevant to prostate cancer progression and five housekeeping genes for normalization (AZGP1, KLK2, SRD5A2, RAM13C, FLNC, GSN, TPM2, GSTM2, TPX2, BGN, COL1A1, SFRP4, ARF1, ATP5E, CLTC, GPS1, PGK1). The signature includes genes mirroring androgen signaling, basal epithelia, stress response, stromal response and also proliferation. ${ }^{125}$ This signature was destilled from 727 initial candidate genes, of which 288 were predictive in the highest and the primary Gleason pattern in prostatectomy cohort. The number of these genes was further reduced by validation in an independent biopsy cohort to finally results in the Genomic Prostate Score (GPS). The assay construction thus incorporates tumor heterogeneity and it delivers a significant prediction of adverse pathology at radical prostatectomy (odds ratio 2.1 per 20 GPS units) in a multivariate model. More so, the GPS showed additional prognostic value in all AUA risk groups. This assay has prior to this been validated to work reliably on small biopsies with only $1 \mathrm{~mm}$ of tumor infiltrate. ${ }^{126}$ The prognostic power of the GPS score was also confirmed by Cullen et al in a cohort of 431 men who underwent radical prostatectomy following has biopsy diagnostis of very low to intermediate risk prostate cancer. Overall, $15 \%$ of men experienced biochemical recurrance (BCR) and 163 had adverse pathology (AP) at radical prostatectomy. The GPS could significantly predict BCR and AP from the biopsy specimens and the authors conclude that this may help to make treatment decisions. ${ }^{127}$ Again, a prospective validation of the GPS score has not been performed yet.

\section{Decipher}

The Decipher signature consists of 22 markers (LASP1, IQGAP3, NFIB, S1PR4, THBS2, ANO7, non-coding transcript, PCDH7, MYBC1, intronic sequence, EPPK1, TSBP, PBX1, NUSAP1, ZWILCH, UBE2C, coding antisense, CAMK2N1, RABGAP1, PCAT-32, GLYATL1P4/PCAT-80, TNFRSF19) that were distilled from an earlier study and that were complemented by non-coding and intronic sequences. ${ }^{128,129}$ The resultant 'genomic classifier'
(GC) was trained to predict disease progression/early metastasis following prostatectomy and was successfully validated as an independent prognosticator of aggressive prostate cancer. ${ }^{128}$ The GC signature has been extensively validated in multiple retrospective studies that confirmed that it can predict prostate cancer metastasis and prostate cancer-specific mortality and hence it may recommend early intensified therapy for high-risk patients after radical prostatectomy to reduce metastasis rate and to improve survival (reviewed in ${ }^{130}$ ). Klein et al ${ }^{131}$ also demonstrated the applicability to needle biopsies in a small cohort $(n=57)$. More lately, the ability to predict prostate cancer-specific mortality from initial needle biopsies was confirmed in a larger set of patients that either underwent surgery or radiation therapy $(n=235) .{ }^{132}$ An ongoing large-scale prospective study of radical prostatectomy patients $(n=2342)$ demonstrated a positive correlation of GC with Gleason scores, pT category, and patient age; however, with significant re-classifications of tumor aggressiveness compared to clinical classifiers. ${ }^{133}$ Especially in the low risk group of patients with Gleason score $3+3=6,20 \%$ were found to have intermediate or even high-risk GC scores. Long-term follow-up will be crucial to clarify the significance of this finding. Another important side-observation of this study was that retrospective central review of Gleason Score 6 cases re-classified Gleason scores in $49 \%$, identifying minor components of Gleason pattern 4 in these cases. ${ }^{134}$ In a large series $(n=561)$ of high-risk cases with adverse pathology on radical prostatectomy, the GC was found to predict prostate cancer-specific mortality independently of CAPRA scores, which underscores the ability of this test to recognize the aggressiveness of this tumor. ${ }^{135}$ Even though it appears, likely, that the GC may aid in the selection of patients suitable for active surveillance, a prospective trial has not been perfomed yet.

\section{Molecular testing-outlook}

Now, which test is best suited for the initial estimation of tumor aggressiveness? Which test is best to identify patients suitable for active surveillance? Which test is best to predict outcome following a specific therapeutic strategy, surgery or radiation therapy? Apparently, all them work well in retrospective studies and it is likely that a prospective randomized validation would show interesting results of clinical impact, even if slightly less impressive than anticipated. So far, there is no data prospectively collected for active surveillance patients according to these test results, which would be the ultimate confirmation. This will require larger cohorts and time, as prostate cancer is progressing so slowly. Comparative studies of these molecular tests would be interesting and there is very little data on this. In breast cancer competing prognostic transcript 
signatures have been compared and showed overlapping but also relevant discordant results. ${ }^{136,137}$ Wei et al profiled four prostate cancer cases in detail analyzing different areas of the tumor. They found significant intratumoral diversity of gene expression, especially in their models of the three aforementioned prognostic signatures. A minor caveat is, that the GC score includes proprietary (non-coding) sequences, that were not included and also their cases were non-low risk cases, which might impact their findings. ${ }^{138}$

It is obvious, that the impact of tumor heterogeneity would need to be considered in judging the value of molecular testing in the individual case. Can a molecular test compensate for insufficient sampling? And finally: facing the enormous costs of these tests, which exceed by far the usual expenses for histology, immunohistochemistry or ordinary molecular testing, we have to ascertain their superior benefit for patient care. So far, it remains to be clarified if a second biopsy with conventional histology or a reference pathology to verify tumor extent and Gleason scores might not be a cost effective alternative to molecular testing. Also, the future impact of multiparametric MRI scanning on therapy planning of prostate cancer is difficult to estimate at the moment.

Many more studies are needed to address these open questions and this has also been outlined in the section of molecular testing of prostate cancer in the recently updated WHO book. ${ }^{139}$ Next to standardization of histopathological diagnostic categories, tumor grading and standards of reporting, additional molecular testing in prostate cancer will gradually evolve, but will require time to be fully implemented.

\section{Disclosure/conflict of interest}

The author declares no conflict of interest.

\section{References}

1 Siegel RL, Miller KD, Jemal A. Cancer statistics, 2017. CA Cancer J Clin 2017;67:7-30.

2 Schultz M, Parzinger H, Posdnjakov DV, et al. Oldest known case of metastasizing prostate carcinoma diagnosed in the skeleton of a 2,700-year-old Scythian king from Arzhan (Siberia, Russia). Int J Cancer 2007;121:2591-2595.

3 Schroder FH, Hugosson J, Roobol MJ, et al. Screening and prostate cancer mortality: results of the European Randomised Study of Screening for Prostate Cancer (ERSPC) at 13 years of follow-up. Lancet 2014;384: 2027-2035.

4 Andriole GL, Crawford ED, Grubb RL 3rd, et al. Mortality results from a randomized prostate-cancer screening trial. N Engl J Med 2009;360:1310-1319.

5 Wilt TJ, Jones KM, Barry MJ, et al. Follow-up of prostatectomy versus observation for early prostate cancer. N Engl J Med 2017;377:132-142.
6 Kesch C, Schutz V, Dieffenbacher S, et al. Multiparametric MRI fusion-guided biopsy for the diagnosis of prostate cancer. Curr Opin Urol; advance online publication, 27 October 2017; doi: 10.1097/ MOU.0000000000000461 [e-pub ahead of print].

7 Verma S, Choyke PL, Eberhardt SC, et al. The current state of MR imaging-targeted biopsy techniques for detection of prostate cancer. Radiology 2017;285: 343-356.

8 Totten RS, Heinemann MW, Hudson PB, et al. Microscopic differential diagnosis of latent carcinoma of prostate. AMA Arch Pathol 1953;55:131-141.

9 Brawer MK, Peehl DM, Stamey TA, et al. Keratin immunoreactivity in the benign and neoplastic human prostate. Cancer Res 1985;45:3663-3667.

10 Hedrick L, Epstein JI. Use of keratin 903 as an adjunct in the diagnosis of prostate carcinoma. Am J Surg Pathol 1989;13:389-396.

11 Signoretti S, Waltregny D, Dilks J, et al. p63 is a prostate basal cell marker and is required for prostate development. Am J Pathol 2000;157:1769-1775.

12 Kristiansen G. [Immunohistochemical algorithms in prostate diagnostics: what's new?]. Pathologe 2009;30 (Suppl 2):146-153.

13 Sailer V, Stephan C, Wernert N, et al. Comparison of p40 (DeltaNp63) and p63 expression in prostate tissues-which one is the superior diagnostic marker for basal cells? Histopathology 2013;63:50-56.

14 Uchida K, Ross H, Lotan T, et al. DeltaNp63 (p40) expression in prostatic adenocarcinoma with diffuse p63 positivity. Hum Pathol 2015;46:384-389.

15 Osunkoya AO, Hansel DE, Sun X, et al. Aberrant diffuse expression of p63 in adenocarcinoma of the prostate on needle biopsy and radical prostatectomy: report of 21 cases. Am J Surg Pathol 2008;32:461-467.

$16 \mathrm{Xu}$ J, Stolk JA, Zhang $\mathrm{X}$, et al. Identification of differentially expressed genes in human prostate cancer using subtraction and microarray. Cancer Res 2000;60:1677-1682.

17 Jiang Z, Woda BA, Rock KL, et al. P504S: a new molecular marker for the detection of prostate carcinoma. Am J Surg Pathol 2001;25:1397-1404.

18 Luo J, Zha S, Gage WR, et al. Alpha-methylacyl-CoA racemase: a new molecular marker for prostate cancer. Cancer Res 2002;62:2220-2226.

19 Magi-Galluzzi C, Luo J, Isaacs WB, et al. Alphamethylacyl-CoA racemase: a variably sensitive immunohistochemical marker for the diagnosis of small prostate cancer foci on needle biopsy. Am J Surg Pathol 2003;27:1128-1133.

20 Herawi M, Parwani AV, Irie J, et al. Small glandular proliferations on needle biopsies: most common benign mimickers of prostatic adenocarcinoma sent in for expert second opinion. Am J Surg Pathol 2005;29:874-880.

$21 \mathrm{Wu}$ CL, Yang XJ, Tretiakova M, et al. Analysis of alpha-methylacyl-CoA racemase (P504S) expression in high-grade prostatic intraepithelial neoplasia. Hum Pathol 2004;35:1008-1013.

22 Zhou M, Chinnaiyan AM, Kleer CG, et al. AlphaMethylacyl-CoA racemase: a novel tumor marker over-expressed in several human cancers and their precursor lesions. Am J Surg Pathol 2002;26:926-931.

23 Jiang Z, Fanger GR, Woda BA, et al. Expression of alpha-methylacyl-CoA racemase (P504s) in various malignant neoplasms and normal tissues: astudy of 761 cases. Hum Pathol 2003;34:792-796. 
24 Tretiakova MS, Sahoo S, Takahashi M, et al. Expression of alpha-methylacyl-CoA racemase in papillary renal cell carcinoma. Am J Surg Pathol 2004;28: 69-76.

25 Gunia S, May M, Scholmann K, et al. Expression of alpha-methylacyl-CoA racemase correlates with histopathologic grading in noninvasive bladder cancer. Virchows Arch 2008;453:165-170.

26 Tischler V, Fritzsche FR, Gerhardt J, et al. Comparison of the diagnostic value of fatty acid synthase (FASN) with alpha-methylacyl-CoA racemase (AMACR) as prostatic cancer tissue marker. Histopathology 2010;56:811-815.

27 Kristiansen G, Fritzsche FR, Wassermann K, et al. GOLPH2 protein expression as a novel tissue biomarker for prostate cancer: implications for tissue-based diagnostics. Br J Cancer 2008;99:939-948.

28 Wei S, Dunn TA, Isaacs WB, et al. GOLPH2 and MYO6: putative prostate cancer markers localized to the Golgi apparatus. Prostate 2008;68:1387-1395.

29 Li W, Wang X, Li B, et al. Diagnostic significance of overexpression of Golgi membrane protein 1 in prostate cancer. Urology 2012;80:e1-e7.

30 Riener MO, Stenner F, Liewen $\mathrm{H}$, et al. Golgi phosphoprotein 2 (GOLPH2) expression in liver tumors and its value as a serum marker in hepatocellular carcinomas. Hepatology 2009;49:1602-1609.

31 Fritzsche FR, Riener MO, Dietel M, et al. GOLPH2 expression in renal cell cancer. BMC Urol 2008;8:15.

32 Fritzsche FR, Kristiansen G, Riener MO, et al. GOLPH2 expression may serve as diagnostic marker in seminomas. BMC Urol 2010;10:4.

33 Berg KD. The prognostic and predictive value of TMPRSS2-ERG gene fusion and ERG protein expression in prostate cancer biopsies. Dan Med J 2016;16:63.

$34 \mathrm{He} \mathrm{H}$, Magi-Galluzzi C, Li J, et al. The diagnostic utility of novel immunohistochemical marker ERG in the workup of prostate biopsies with "atypical glands suspicious for cancer". Am J Surg Pathol 2011;35: 608-614.

35 Lee WH, Morton RA, Epstein JI, et al. Cytidine methylation of regulatory sequences near the piclass glutathione S-transferase gene accompanies human prostatic carcinogenesis. Proc Natl Acad Sci USA 1994;91:11733-11737.

36 Sailer V, Eberhard HL, Stephan C, et al. Glutathione S-transferase-pi protein expression in prostate cancer-not always a useful diagnostic tool. Histopathology 2015;67:577-579.

37 Epstein JI, Egevad L, Humphrey PA, et al. Members of the IIIDUPG. Best practices recommendations in the application of immunohistochemistry in the prostate: report from the International Society of Urologic Pathology consensus conference. Am J Surg Pathol 2014;38:e6-e19.

38 Ablin RJ, Bronson P, Soanes WA, et al. Tissue- and species-specific antigens of normal human prostatic tissue. J Immunol 1970;104:1329-1339.

39 Frankel AE, Rouse RV, Wang MC, et al. Monoclonal antibodies to a human prostate antigen. Cancer Res 1982;42:3714-3718.

40 Bates RJ, Chapman CM, Prout GR Jr., et al. Immunohistochemical identification of prostatic acid phosphatase: correlation of tumor grade with acid phosphatase distribution. J Urol 1982;127:574-580.

41 Torenbeek R, Lagendijk JH, Van Diest PJ, et al. Value of a panel of antibodies to identify the primary origin of adenocarcinomas presenting as bladder carcinoma. Histopathology 1998;32:20-27.

42 Kristiansen I, Stephan C, Jung K et al. Sensitivity of HOXB13 as a diagnostic immunohistochemical marker of prostatic origin in prostate cancer metastases: comparison to PSA, prostein, androgen receptor, ERG, NKX3.1, PSAP, and PSMA. Int J Mol Sci 2017;18: 1151.

43 Kalos M, Askaa J, Hylander BL, et al. Prostein expression is highly restricted to normal and malignant prostate tissues. Prostate 2004;60:246-256.

$44 \mathrm{Xu}$ J, Kalos M, Stolk JA, et al. Identification and characterization of prostein, a novel prostate-specific protein. Cancer Res 2001;61:1563-1568.

45 Yin M, Dhir R, Parwani AV. Diagnostic utility of p501s (prostein) in comparison to prostate specific antigen (PSA) for the detection of metastatic prostatic adenocarcinoma. Diagn Pathol 2007;2:41.

46 Sheridan T, Herawi M, Epstein JI, et al. The role of P501S and PSA in the diagnosis of metastatic adenocarcinoma of the prostate. Am J Surg Pathol 2007;31:1351-1355.

47 He WW, Sciavolino PJ, Wing J, et al. A novel human prostate-specific, androgen-regulated homeobox gene (NKX3.1) that maps to $8 \mathrm{p} 21$, a region frequently deleted in prostate cancer. Genomics 1997;43:69-77.

48 Bowen C, Bubendorf L, Voeller HJ, et al. Loss of NKX3.1 expression in human prostate cancers correlates with tumor progression. Cancer Res 2000;60: 6111-6115.

49 Queisser A, Hagedorn SA, Braun M, et al. Comparison of different prostatic markers in lymph node and distant metastases of prostate cancer. Mod Pathol 2015;28:138-145.

50 Varinot J, Cussenot O, Roupret M, et al. HOXB13 is a sensitive and specific marker of prostate cells, useful in distinguishing between carcinomas of prostatic and urothelial origin. Virchows Arch 2013;463:803-809.

51 Zabalza CV, Adam M, Burdelski C, et al. HOXB13 overexpression is an independent predictor of early PSA recurrence in prostate cancer treated by radical prostatectomy. Oncotarget 2015;6:12822-12834.

52 Barresi V, Ieni A, Cardia R, et al. HOXB13 as an immunohistochemical marker of prostatic origin in metastatic tumors. APMIS 2016;124:188-193.

53 Varinot J, Furudoi A, Drouin S, et al. HOXB13 protein expression in metastatic lesions is a promising marker for prostate origin. Virchows Arch 2016;468: 619-622.

54 Edwards S, Campbell C, Flohr P, et al. Expression analysis onto microarrays of randomly selected cDNA clones highlights HOXB13 as a marker of human prostate cancer. Br J Cancer 2005;92:376-381.

55 Huber F, Montani M, Sulser T, et al. Comprehensive validation of published immunohistochemical prognostic biomarkers of prostate cancer -what has gone wrong? A blueprint for the way forward in biomarker studies. Br J Cancer 2015;112:140-148.

56 Scholzen T, Gerdes J. The Ki-67 protein: from the known and the unknown. J Cell Physiol 2000;182: 311-322.

57 Kristiansen G. Diagnostic and prognostic molecular biomarkers for prostate cancer. Histopathology 2012;60:125-141.

58 Sekula P, Mallett S, Altman DG, et al. Did the reporting of prognostic studies of tumour markers improve since the introduction of REMARK guideline? 
A comparison of reporting in published articles. PLoS ONE 2017;12:e0178531.

59 McShane LM, Altman DG, Sauerbrei W, et al. Reporting recommendations for tumor marker prognostic studies (REMARK). J Natl Cancer Inst 2005;97: 1180-1184.

60 Polley MY, Leung SC, McShane LM, et al. An international Ki67 reproducibility study. J Natl Cancer Inst 2013;105:1897-1906.

61 Christgen M, von Ahsen S, Christgen $\mathrm{H}$, et al. The region-of-interest size impacts on Ki67 quantification by computer-assisted image analysis in breast cancer. Hum Pathol 2015;46:1341-1349.

62 Denkert C, Budczies J, von Minckwitz G, et al. Strategies for developing Ki67 as a useful biomarker in breast cancer. Breast 2015;24(Suppl 2):S67-S72.

63 Goltz D, Montani M, Braun M, et al. Prognostic relevance of proliferation markers (Ki-67, PHH3) within the cross-relation of ERG translocation and androgen receptor expression in prostate cancer. Pathology 2015;47:629-636.

64 Deocampo ND, Huang H, Tindall DJ. The role of PTEN in the progression and survival of prostate cancer. Minerva Endocrinol 2003;28: 145-153.

65 Carver BS, Tran J, Gopalan A, et al. Aberrant ERG expression cooperates with loss of PTEN to promote cancer progression in the prostate. Nat Genet 2009;41: 619-624.

66 King JC, Xu J, Wongvipat J, et al. Cooperativity of TMPRSS2-ERG with PI3-kinase pathway activation in prostate oncogenesis. Nat Genet 2009;41:524-526.

67 Krohn A, Diedler T, Burkhardt L, et al. Genomic deletion of PTEN is associated with tumor progression and early PSA recurrence in ERG fusion-positive and fusion-negative prostate cancer. Am J Pathol 2012;181:401-412.

68 McMenamin ME, Soung P, Perera S, et al. Loss of PTEN expression in paraffin-embedded primary prostate cancer correlates with high Gleason score and advanced stage. Cancer Res 1999;59:4291-4296.

69 Halvorsen OJ, Haukaas SA, Akslen LA. Combined loss of PTEN and p27 expression is associated with tumor cell proliferation by Ki-67 and increased risk of recurrent disease in localized prostate cancer. Clin Cancer Res 2003;9:1474-1479.

70 Schmitz M, Grignard G, Margue C, et al. Complete loss of PTEN expression as a possible early prognostic marker for prostate cancer metastasis. Int J Cancer 2007;120:1284-1292.

71 Cuzick J, Yang ZH, Fisher G, et al. Prognostic value of PTEN loss in men with conservatively managed localised prostate cancer. Br J Cancer 2013;108: 2582-2589.

72 Lotan TL, Heumann A, Rico SD, et al. PTEN loss detection in prostate cancer: comparison of PTEN immunohistochemistry and PTEN FISH in a large retrospective prostatectomy cohort. Oncotarget 2017;8:65566-65576.

73 Trock BJ, Fedor H, Gurel B, et al. PTEN loss and chromosome 8 alterations in Gleason grade 3 prostate cancer cores predicts the presence of un-sampled grade 4 tumor: implications for active surveillance. Mod Pathol 2016;29:764-771.

74 Cyll K, Ersvaer E, Vlatkovic L, et al. Tumour heterogeneity poses a significant challenge to cancer biomarker research. Br J Cancer 2017;117:367-375.
75 Ferraldeschi R, Nava Rodrigues D, Riisnaes R, et al. PTEN protein loss and clinical outcome from castration-resistant prostate cancer treated with abiraterone acetate. Eur Urol 2015;67:795-802.

76 Tomlins SA, Rhodes DR, Perner S, et al. Recurrent fusion of TMPRSS2 and ETS transcription factor genes in prostate cancer. Science 2005;310:644-648.

77 Demichelis F, Fall K, Perner S, et al. TMPRSS2:ERG gene fusion associated with lethal prostate cancer in a watchful waiting cohort. Oncogene 2007;26: 4596-4599.

78 Attard G, Clark J, Ambroisine L, et al. Duplication of the fusion of TMPRSS2 to ERG sequences identifies fatal human prostate cancer. Oncogene 2008;27: 253-263.

79 Berg KD, Vainer B, Thomsen FB, et al. ERG protein expression in diagnostic specimens is associated with increased risk of progression during active surveillance for prostate cancer. Eur Urol 2014;66:851-860.

80 FitzGerald LM, Agalliu I, Johnson K, et al. Association of TMPRSS2-ERG gene fusion with clinical characteristics and outcomes: results from a population-based study of prostate cancer. BMC Cancer 2008;8:230.

81 Esgueva R, Perner S, JL C, et al. Prevalence of TMPRSS2-ERG and SLC45A3-ERG gene fusions in a large prostatectomy cohort. Mod Pathol 2010;23: 539-546.

82 Minner S, Enodien M, Sirma H, et al. ERG status is unrelated to PSA recurrence in radically operated prostate cancer in the absence of antihormonal therapy. Clin Cancer Res 2011;17:5878-5888.

83 Pettersson A, Graff RE, Bauer SR, et al. The TMPRSS2:ERG rearrangement, ERG expression, and prostate cancer outcomes: a cohort study and metaanalysis. Cancer Epidemiol Biomark Prev 2012;21: 1497-1509.

84 Hoogland AM, Jenster G, van Weerden WM, et al. ERG immunohistochemistry is not predictive for PSA recurrence, local recurrence or overall survival after radical prostatectomy for prostate cancer. Mod Pathol 2012;25:471-479.

85 Minner S, Gartner M, Freudenthaler F, et al. Marked heterogeneity of ERG expression in large primary prostate cancers. Mod Pathol 2013;26:106-116.

86 Ahearn TU, Pettersson A, Ebot EM, et al. A prospective investigation of PTEN loss and ERG expression in lethal prostate cancer. J Natl Cancer Inst 2016;108: pii: djv346.

87 Mateo J, Carreira S, Sandhu S, et al. DNA-repair defects and olaparib in metastatic prostate cancer. N Engl J Med 2015;373:1697-1708.

88 Jeronimo C, Bastian PJ, Bjartell A, et al. Epigenetics in prostate cancer: biologic and clinical relevance. Eur Urol 2011;60:753-766.

89 Bastian PJ, Ellinger J, Wellmann A, et al. Diagnostic and prognostic information in prostate cancer with the help of a small set of hypermethylated gene loci. Clin Cancer Res 2005;11:4097-4106.

90 Holmes EE, Jung M, Meller S, et al. Performance evaluation of kits for bisulfite-conversion of DNA from tissues, cell lines, FFPE tissues, aspirates, lavages, effusions, plasma, serum, and urine. PLoS ONE 2014;9:e93933.

91 Vanaja DK, Ehrich M, Van den Boom D, et al. Hypermethylation of genes for diagnosis and risk stratification of prostate cancer. Cancer Invest 2009;27:549-560. 
92 Weiss G, Cottrell S, Distler J, et al. DNA methylation of the PITX2 gene promoter region is a strong independent prognostic marker of biochemical recurrence in patients with prostate cancer after radical prostatectomy. J Urol 2009;181:1678-1685.

93 Banez LL, Sun L, van Leenders GJ, et al. Multicenter clinical validation of PITX2 methylation as a prostate specific antigen recurrence predictor in patients with post-radical prostatectomy prostate cancer. J Urol 2010;184:149-156.

94 Dietrich D, Hasinger O, Banez LL, et al. Development and clinical validation of a real-time PCR assay for PITX2 DNA methylation to predict prostate-specific antigen recurrence in prostate cancer patients following radical prostatectomy. J Mol Diagn 2013;15: 270-279.

95 Litovkin K, Joniau S, Lerut E, et al. Methylation of PITX2, HOXD3, RASSF1 and TDRD1 predicts biochemical recurrence in high-risk prostate cancer. J Cancer Res Clin Oncol 2014;140:1849-1861.

96 Vasiljevic N, Ahmad AS, Carter PD, et al. DNA methylation of PITX2 predicts poor survival in men with prostate cancer. Biomark Med 2014;8: 1143-1150.

97 Luan ZM, Zhang H, Qu XL. Prediction efficiency of PITX2 DNA methylation for prostate cancer survival. Genet Mol Res; advance online publication, 25 April 2016;15; doi: 10.4238/gmr.15026750.

98 Ahmad AS, Vasiljevic N, Carter P, et al. A novel DNA methylation score accurately predicts death from prostate cancer in men with low to intermediate clinical risk factors. Oncotarget 2016;7:71833-71840.

99 Uhl B, Gevensleben H, Tolkach Y, et al. PITX2 DNA methylation as biomarker for individualized risk assessment of prostate cancer in core biopsies. J Mol Diagn 2017;19:107-114.

100 Capitanio U, Pfister D, Emberton M. Repeat prostate biopsy: rationale, indications, and strategies. Eur Urol Focus 2015;1:127-136.

101 Woodrum DA, Kawashima A, Gorny KR, et al. Prostate cancer: state of the art imaging and focal treatment. Clin Radiol 2017;72:665-679.

102 Sailer V, Kristiansen G. Histopathological screening for prostate carcinoma: is a benign biopsy a negative biopsy? APMIS 2014;122:690-698.

103 Trock BJ, Brotzman MJ, Mangold LA, et al. Evaluation of GSTP1 and APC methylation as indicators for repeat biopsy in a high-risk cohort of men with negative initial prostate biopsies. BJU Int 2012;110:56-62.

104 Stewart GD, Van Neste L, Delvenne P, et al. Clinical utility of an epigenetic assay to detect occult prostate cancer in histopathologically negative biopsies: results of the MATLOC study. J Urol 2013;189:1110-1116.

105 Partin AW, Van Neste L, Klein EA, et al. Clinical validation of an epigenetic assay to predict negative histopathological results in repeat prostate biopsies. J Urol 2014;192:1081-1087.

106 Singh D, Febbo PG, Ross K, et al. Gene expression correlates of clinical prostate cancer behavior. Cancer Cell 2002;1:203-209.

107 Dhanasekaran SM, Barrette TR, Ghosh D, et al. Delineation of prognostic biomarkers in prostate cancer. Nature 2001;412:822-826.

108 Henshall SM, Afar DE, Hiller J, et al. Survival analysis of genome-wide gene expression profiles of prostate cancers identifies new prognostic targets of disease relapse. Cancer Res 2003;63:4196-4203.
109 Kristiansen G, Pilarsky C, Wissmann C, et al. Expression profiling of microdissected matched prostate cancer samples reveals CD166/MEMD and CD24 as new prognostic markers for patient survival. J Pathol 2005;205:359-376.

110 Peng Z, Skoog L, Hellborg H, et al. An expression signature at diagnosis to estimate prostate cancer patients' overall survival. Prostate Cancer Prostatic Dis 2014;17:81-90.

111 Glinsky GV, Berezovska O, Glinskii AB. Microarray analysis identifies a death-from-cancer signature predicting therapy failure in patients with multiple types of cancer. J Clin Invest 2005;115:1503-1521.

112 Lapointe J, Li C, Higgins JP, et al. Gene expression profiling identifies clinically relevant subtypes of prostate cancer. Proc Natl Acad Sci USA 2004;101: 811-816.

113 Sboner A, Demichelis F, Calza S, et al. Molecular sampling of prostate cancer: a dilemma for predicting disease progression. BMC Med Genomics 2010;3:8.

114 Ohl F, Jung M, Xu C, et al. Gene expression studies in prostate cancer tissue: which reference gene should be selected for normalization? J Mol Med 2005;83: 1014-1024.

115 Kretschmer A, Tolkach Y, Ellinger J, et al. Molecular biomarkers and prognostic factors for prostate cancer. Der Urologe Ausg A 2017;56:933-944.

116 Hanahan D, Weinberg RA. The hallmarks of cancer. Cell 2000;100:57-70.

117 Cuzick J, Swanson GP, Fisher G, et al. Prognostic value of an RNA expression signature derived from cell cycle proliferation genes in patients with prostate cancer: a retrospective study. Lancet Oncol 2011;12:245-255.

118 Cuzick J, Berney DM, Fisher G, et al. Prognostic value of a cell cycle progression signature for prostate cancer death in a conservatively managed needle biopsy cohort. Br J Cancer 2012;106:1095-1099.

119 Cuzick J, Stone S, Fisher G, et al. Validation of an RNA cell cycle progression score for predicting death from prostate cancer in a conservatively managed needle biopsy cohort. Br J Cancer 2015;113: 382-389.

120 Cooperberg MR, Simko JP, Cowan JE, et al. Validation of a cell-cycle progression gene panel to improve risk stratification in a contemporary prostatectomy cohort. J Clin Oncol 2013;31:1428-1434.

121 Freedland SJ, Gerber L, Reid J, et al. Prognostic utility of cell cycle progression score in men with prostate cancer after primary external beam radiation therapy. Int J Radiat Oncol Biol Phys 2013;86:848-853.

122 Sommariva S, Tarricone R, Lazzeri M, et al. Prognostic value of the cell cycle progression score in patients with prostate cancer: a systematic review and meta-analysis. Eur Urol 2016;69:107-115.

123 Oderda M, Cozzi G, Daniele L, et al. Cell-cycle progression-score might improve the current risk assessment in newly diagnosed prostate cancer patients. Urology 2017;102:73-78.

124 Tosoian JJ, Chappidi MR, Bishoff JT, et al. Prognostic utility of biopsy-derived cell cycle progression score in patients with National Comprehensive Cancer Network low-risk prostate cancer undergoing radical prostatectomy: implications for treatment guidance. BJU Int; advance online publication 8 May 2017;. doi: 10.1111/bju.1391 [e-pub ahead of print].

125 Klein EA, Cooperberg MR, Magi-Galluzzi C, et al. A 17gene assay to predict prostate cancer aggressiveness in 
the context of Gleason grade heterogeneity, tumor multifocality, and biopsy undersampling. Eur Urol 2014;66:550-560.

126 Knezevic D, Goddard AD, Natraj N, et al. Analytical validation of the Oncotype DX prostate cancer assay a clinical RT-PCR assay optimized for prostate needle biopsies. BMC Genom 2013;14:690.

127 Cullen J, Rosner IL, Brand TC, et al. A biopsy-based 17-gene genomic prostate score predicts recurrence after radical prostatectomy and adverse surgical pathology in a racially diverse population of men with clinically low- and intermediate-risk prostate cancer. Eur Urol 2015;68:123-131.

128 Erho N, Crisan A, Vergara IA, et al. Discovery and validation of a prostate cancer genomic classifier that predicts early metastasis following radical prostatectomy. PLoS ONE 2013;8:e66855.

129 Nakagawa T, Kollmeyer TM, Morlan BW, et al. A tissue biomarker panel predicting systemic progression after PSA recurrence post-definitive prostate cancer therapy. PLoS ONE 2008;3:e2318.

$130 \mathrm{Na}$ R, Wu Y, Ding Q, et al. Clinically available RNA profiling tests of prostate tumors: utility and comparison. Asian J Androl 2016;18:575-579.

131 Klein EA, Haddad Z, Yousefi K, et al. Decipher genomic classifier measured on prostate biopsy predicts metastasis risk. Urology 2016;90:148-152.

132 Nguyen PL, Haddad Z, Ross AE, et al. Ability of a genomic classifier to predict metastasis and prostate cancer-specific mortality after radiation or surgery based on needle biopsy specimens. Eur Urol 2017;72:845-852.
133 Den RB, Santiago-Jimenez M, Alter J, et al. Decipher correlation patterns post prostatectomy: initial experience from 2342 prospective patients. Prostate Cancer Prostat Dis 2016;19:374-379.

134 Klein EA, Santiago-Jimenez M, Yousefi K, et al. Molecular analysis of low grade prostate cancer using a genomic classifier of metastatic potential. J Urol 2017;197:122-128.

135 Karnes RJ, Choeurng V, Ross AE, et al. Validation of a genomic risk classifier to predict prostate cancerspecific mortality in men with adverse pathologic features. Eur Urol 2017; pii: S0302-2838(17)30264-6.

136 Varga Z, Sinn P, Fritzsche F, et al. Comparison of EndoPredict and Oncotype DX test results in hormone receptor positive invasive breast cancer. PLoS ONE 2013;8:e58483.

137 Buus R, Sestak I, Kronenwett R, et al. Comparison of EndoPredict and EPclin with Oncotype DX recurrence score for prediction of risk of distant recurrence after endocrine therapy. J Natl Cancer Inst; advance online publication, 10 July 2016; 108; doi: 10.1093/jnci/ djw149.

138 Wei L, Wang J, Lampert E, et al. Intratumoral and intertumoral genomic heterogeneity of multifocal localized prostate cancer impacts molecular classifications and genomic prognosticators. Eur Urol 2017;71:183-192.

139 Moch H, Humphrey PA, Ulbright TM, Reuter VE, (Eds.): WHO Classification of Tumours of the Urinary System and Male Genital Organs (4th edition). IARC: Lyon 2016. 